\title{
ON THE LOCAL DIFFEOMORPHISM AND SUBMANIFOLDS OF MATRICES WITH FIXED JORDAN BLOCK STRUCTURE
}

\section{ALEXANDER BONDAR AND YAKOV DYMARSKII}

Abstract. In this paper a local diffeomorphism, which allows to investigate different matrix submanifolds is introduced. With its help the submanifold of complex matrices with fixed structure of Jordan blocks is described. Then, explicit formulas of some special perturbations of Jordan structure are given.

Mathematics subject classification (2010): 15B99, 53B25.

Keywords and phrases: Submanifolds of matrices, Jordan form, perturbation.

\section{REFERENCES}

[1] V. I. Arnold, Modes and quasimodes, Funct. Anal. Appl. 6, 2 (1972), 94-101.

[2] D. Fujiwara, M. Tanikawa And Sh. Yukita, The spectrum of Laplasian I, Proc. Jap. Acad. Ser. A. 54, 4 (1978), 87-91.

[3] YA. M. DYMARSKII, Manifold method in the eigenvector theory of nonlinear operators, J. Math. Sci. 154, 5 (2008), 655-815.

[4] YA. M. DYMARSKII, Local research of manifolds generated by families of self-adjoint operators, Topology 48 (2009), 213-223.

[5] V. I. ARnold, On matrices depending on parameters, Russ. Math. Sur. 26, 2:29 (1971).

[6] F. Riesz, B. Sz.-NAGy, Functional Analysis, Dover Publ., 1990.

[7] F. R. Gantmakher, The theory of matrices, American Mathematical Soc., 1959.

[8] A. A. BONDAR, YA. M. DYMARSKII, Submanifolds of compact operators with fixed multiplicities of eigenvalues, Ukrainian Mathematical Journal 63, 9 (2012), 1349-1360. 